\title{
REPRESENTACIONES CONTEMPORÁNEAS DE LA ESCLAVITUD EN LAS MUJERES AFROCOSTARRICENSES: Desde sus propias voces
}

\section{CONTEMPORARY REPRESENTATIONS OF SLAVERY IN AFRO-COSTA RICAN WOMEN: From their own voices}

\author{
Evelyn Castro Carmiol
}

\begin{abstract}
RESUMEN
El estudio comprende las formas contemporáneas en que la esclavitud como mentalidad se reproduce en el imaginario social costarricense, a partir de lo cual se continúan dando acciones concretas de discriminación y opresión por el género y la raza hacia las mujeres afrocostarricenses; asimismo, se retoman las diferentes estrategias que ellas utilizan para resistirse, relacionando su situación actual con las vivencias de sus ancestras.
\end{abstract}

PALABRAS CLAVE: COSTA RICA * MUJERES * NEGRAS * DISCRIMINACIÓN RACIAL * ESCLAVITUD $*$ COMPORTAMIENTO

\section{ABSTRACT}

This study encompasses contemporary forms by which slavery, as a mentality, is reproduced in the Costa Rican social imaginary. Based on this concept, several acts of discrimination and oppression by their gender and race are still occurring against Afro-Costa Rican women. Also, different strategies used to resist those events are recalled from their life experiences, linking their current situation with the experiences of their female ancestors.

KEY WORDS: COSTA RICA * WOMEN * BLACKS * RACIAL DISCRIMINATION * SLAVERY * BEHAVIOUR

Licenciada en Trabajo Social, Universidad de Costa Rica, Máster en Estudios de Género y Cultura. Mención de Ciencias Sociales, Universidad de Chile.

evelyncastrocar@yahoo.com 


\section{INTRODUCCIÓN}

Las personas desde que nacen son clasificadas en el imaginario social en dos categorías básicas: hombre o mujer, siendo construcciones sociales que determinan lo que se espera de las personas a partir de sus cuerpos físicos. Al respecto Lamas citando a Scott plantea que "la diferencia de sexos es una forma primaria de diferenciación significativa" (1996: 330), es decir la ubicación y el trato social que las personas reciben están determinadas en primer instancia por el sistema sexo-género.

$\mathrm{Al}$ prevalecer una estructura social basada en el patriarcado, "lo femenino" se ha ubicado en una categoría inferior con relación a lo considerado como "masculino", por ende las relaciones sociales han estado mediadas por estas ideas pudiéndose afirmar que "el género se constituye en la forma primaria de relaciones significantes de poder" (Ibíd: 330).

Además, desde el mismo cuerpo físico de las personas se representan otras características que cobran un significado social, siendo el género transversal en la medida que se feminizan o no. Es ahí donde se denota la raza como una categoría a la que se le ha otorgado un valor social en tanto ha prevalecido el significado de lo blanco como dominante de diferentes maneras a través de la historia. Desde el cuerpo físico por medio del cual las personas se presentan ante el mundo, las mismas son objeto de simbolizaciones $y$ tratos sociales desiguales por el género y la raza a la que pertenezcan.

En relación con lo anterior, interesa considerar las vivencias de las mujeres negras en tanto son categorizadas a partir de sus cuerpos "femeninos y negros" como objetos de simbolizaciones $y$ acciones negativas por parte de otros en ubicaciones de poder reconocidas frente a ellas. A partir de esto cabe la reflexión sobre la manera en que las mujeres negras han logrado sobrevivir en un sistema patriarcal y occidentalizado, lo cual implica realizar una contextualización histórica y entender su situación concreta, actualmente, como una continuidad de las vivencias de sus ancestras.

Al respecto, específicamente la esclavitud como sistema socioeconómico se abolió formal- mente, sin embargo, las ideas que la fundamentaron no se han podido abolir en la sociedad de la misma manera, repercutiendo aún en la vida de las mujeres negras. A este imaginario social es lo que se va a denominar como representaciones contemporáneas de la esclavitud, contemplando tanto las formas de opresión y discriminación como las formas de resistencia implementadas específicamente por las mujeres afrocostarricenses.

Como objetivo general de la investigación se planteó:

Conocer la vivencias de discriminación y opresión de género y racial de las mujeres afrocostarricenses dadas por el sistema sexogénero vigente.

Como objetivos específicos se buscó:

Identificar formas de discriminación $y$ opresión actuales que viven las mujeres afrocostarricenses. [Asimismo] Indagar los mecanismos de resistencia de las mujeres afrocostarricenses ante las representaciones de la esclavitud trasmitidas históricamente. [Por último] Dilucidar qué elementos de la esclavitud, como fenómeno histórico y social, se mantienen vigentes en los imaginarios $y$ vivencias de las mujeres afrocostarricenses.

En síntesis esta investigación pretende denotar una realidad histórica vivida por las mujeres negras en Costa Rica y a partir de esta conocer ¿qué situaciones de discriminación y opresión se perpetúan? y ¿qué condiciones nuevas se dan reproduciendo relaciones de poder, así como aquellas estrategias que las mujeres afrocostarricenses han logrado desarrollar como legado de sus ancestras para sobrevivir y progresar, reconstruyendo la misma subjetividad femenina?

\section{EL CUERPO COMO JUSTIFICACIÓN DE LAS DIFERENCIAS SOCIALES}

Como se mencionó anteriormente el cuerpo físico de las personas es la base para el establecimiento de dos categorías básicas: hombre y mujer, en tanto su sexo, a partir de lo 
cual se ha creado una construcción cultural de la diferencia sexual, aludiendo a las relaciones sociales de los sexos (Lamas, 1996: 332), siendo lo que se conoce cómo género.

Según Lamas (1996: 343) reconocer la diferencia de papeles implica una jerarquización, es decir la "naturalización" de las diferencias entre los hombres y las mujeres más allá de las características físicas, ha significado la ubicación de "lo femenino" como una categoría inferior. Al respecto Amorós plantea que "lo femenino ha constituido un cajón de significados mayormente opresores, ya que la mujer como construcción no es un ser autónomo, depende de lo que el hombre defina en relación a él" (1991: 61). De esta manera se ha ubicado lo femenino como la alteridad, para lo cual se han legitimado diferentes manifestaciones de violencia al nivel simbólico y directo.

$\mathrm{Al}$ respecto Bourdieu plantea el concepto de Violencia Simbólica en tanto

La preminencia universalmente reconocida a los hombres se afirma en la objetividad de las estructuras sociales. [Es decir, se establecen] matrices de las percepciones de los pensamientos $y$ de las acciones de todos los miembros de la sociedad, trascendentales históricas que, al ser universalmente reconocidas se imponen a cualquier agente como trascendente (2000: 49).

Este tipo de violencia se manifiesta desde el lugar en el que son situadas las mujeres "lo doméstico", como continuidad "natural" ante su capacidad de reproducción. Consecuentemente se predeterminan las tareas que "deben" asumir, siendo tareas que las encierran y someten al servicio de los otros y limitan sus posibilidades de trascender a funciones para sí (Ibíd: 31). Otras formas de violencia simbólica se dan en la manera en que se espera las mujeres deben re-presentarse ante los demás, incluyendo sus expresiones corporales, los movimientos y gestos, la manera en que se comunican con los demás y hasta en su vestimenta, ante lo cual se crean sanciones sociales en tanto cada mujer se acerque o no a los parámetros permitidos.
También se dan otras representaciones de la violencia hacia las mujeres, por medio de aquellas acciones que de manera directa buscan someterlas. Lo anterior se denomina Violencia Basada en el Género (VBG) siendo

Actos concretos que pueden tener como resultado un daño o sufrimiento físico, social o psicológico para las mujeres, así como las amenazas de tales actos, la coacción o la privación arbitraria de la libertad, tanto si se producen en la vida pública, como en la vida privada (Velzeboer, 2003: 4).

Precisamente en muchas culturas y sociedades se dan prácticas directas de agresión hacia las mujeres, porque desde el imaginario social está legitimada la supuesta inferioridad de las mismas, lo cual en algunos casos se instala $y$ reproduce o se hace omisión a dicha problemática.

$\mathrm{Al}$ igual que el sexo, la raza se inscribe en el cuerpo como un predeterminante externo, visible, a partir del cual se atribuyen características sociales en este caso según el grupo racial en el que se clasifiquen las personas, respondiendo a ideas legitimadas históricamente.

En ese sentido se retomó el concepto de razas sociológicas propuesto por Bastide, quien se refiere a grupos internos o externos respecto a una comunidad, de los cuales se presumen atributos biológicos comunes, siendo el rasgo físico el símbolo de pertenencia a un grupo social separado (1970: 34). Si bien es cierto el trato hacia las personas según el grupo racial al que pertenezca, es relativo a cada lugar, se considera que existen significados sociales que se han generalizado y que se basan en las características físicas de las mismas.

En relación con la población afrodescendiente se puede afirmar que al nivel social se le ha dado un significado negativo a "lo negro", equiparándolo a valores y características negativas, con lo que igualmente se han fundamentado a través de la historia las prácticas de discriminación racial.

Al respecto Campbell define el racismo como: 
Una construcción histórica que ha funcionado como ideología dominante que fundamenta la superioridad racial desde el grupo social y económico con poder y que a su vez es arraigado en el común de las personas (2002: 17-18).

Estas ideas racistas surgen para justificar un sistema socioeconómico en beneficio de grupos con poder, como lo fue la esclavitud, manifestándose de una manera específica hacia las mujeres por el sexismo también imperante.

Se va a entender la esclavitud como "el estado o condición de las personas sobre los que se ejercen todos o parte de los poderes atribuidos al derecho de propiedad" (OACDH, 1956: artículo 7). Aunque la esclavitud como sistema socioeconómico formal se abolió, se han venido dando diferentes prácticas en tanto abusos degradantes hacia las personas, las cuales se han catalogado como prácticas análogas a la esclavitud (OACDH, 1956: artículo 1).

Además de estas prácticas se han mantenido diferenciaciones entre las personas que vienen desde el mismo sistema esclavista colonial, trascendiendo el tiempo por prejuicios vigentes, por ende se puede afirmar que ha prevalecido la esclavitud como una mentalidad (OACDH, s/f.). Bastide (1970) coincide al afirmar que los prejuicios raciales empezaron por un interés económico, aunque se han ido transformando con el tiempo, se han mantenido cambiando solo de ropaje. A estas ideas es a lo que se va a entender en el presente estudio como Representaciones contemporáneas de la esclavitud.

Sobre esto Calvo (2004) plantea que en el caso de las mujeres negras se ha dado el sexismo y el racismo juntos por lo que han sido víctimas potenciales de la esclavitud. Según Acuña (2004) en Costa Rica, las mujeres negras vivieron de manera más rígida el régimen esclavista y ocuparon una posición inferior en la escala social, ya que además de que fueron explotadas económicamente lo fueron en el ámbito sexual.

Se les consideraba como mujeres de la calle, lo cual se dio debido a la misma explotación sexual de sus amos como a la prostitución que las forzaban a ejercer (Acuña, 2004: 7; 2). Asimismo, pasaron de asumir un papel económico central y del goce de derechos en las sociedades africanas, a asumir tareas domésticas y al servicio de los/as demás, una vez que fueron esclavizadas (Acuña, 1999: 3). Aunado a lo anterior, vivieron maltrato físico desde el momento en que fueron embarcadas, así como al nivel emocional a través de amenazas de revenderlas, alejarlas de su familia $y$ descendencia, negándoles la libertad una vez que murieran sus amos, hasta ser controladas en la manera que debían vestir (1999: 3).

\section{FORMAS DE RESISTENCIA}

Sobre estas prácticas de discriminación por la raza y el sexo cabe la reflexión de la forma en que han sido asumidas por las mujeres negras, como alternativa de sobrevivencia para intentar un cambio social. Desde "lo femenino" Amorós (1991) plantea que los hombres han tenido la necesidad de crear prohibiciones de tareas a las mujeres porque se prohíbe lo que se puede hacer, por ende a aquellas estrategias utilizadas por las mujeres para oponerse a condiciones de sometimiento basadas en el género es a lo que se va a entender como las formas de resistencia.

Al respecto Irigaray lo define como mecanismos de derrocamiento, en tanto "la mujer se defina para sí y no siguiendo de manera enajenada los mandatos de la feminidad" (1994: 95).

Para lo anterior, Bourdieu propone que "la alternativa que existe inicia desde la lucha cognitiva sobre el sentido de las cosas del mundo, en especial las realidades sexuales" (2000: 26). Al respecto Tubert coincide al afirmar que "en el corazón de la vida psíquica se encuentra una tenaz resistencia inconsciente de aceptar el modelo de identidad asumida por el yo, lo cual se puede ver como un proceso subsiguiente a la reconstrucción interna de la subjetividad" (1995: 5).

Otra forma de resistencia es abordada por Irigaray (1994) quien afirma que para que las mujeres sean valoradas como sujetas, se debe considerar un cambio desde el sistema de símbolos que son el fundamento de las prácticas cotidianas, lo que representa un cambio de los discursos. Al respecto Tubert (1995) coincide al afirmar que se debe proporcionar 
un espacio para la palabra de las mujeres, que permite construir representaciones discursivas diferentes esto por cuanto desde la palabra de las mujeres se debilita el discurso oficial.

Por otro lado Arendt retoma la importancia de "pensar en lo que hacemos" (2001: 18), con lo cual se trasciende a la acción. $\mathrm{Al}$ respecto afirma que "las personas desarrollan la capacidad que le es propia, que es la capacidad de ser libre. Esa libertad no implica solo tomar decisiones sino trascender lo dado y empezar algo nuevo, así se trasciende la naturaleza cuando se actúa” (2001: VIII).

De Beauvoir retoma el concepto de libertad en la medida que las mujeres como individuos trasciendan a un proyecto, alcanzando la libertad por medio de su perpetuo avance hacia otras libertades (1990: 30-31). Con esto la autora enfatiza en la posibilidad de desafiar los mandatos de género buscando la independencia aún desde una posición de dependencia asignada.

Por último se retoma la posibilidad de la organización colectiva desde las mujeres, una vez que se realiza un proceso de derrocamiento individual y se trasciende a la búsqueda de la realización personal, se aboga por la posibilidad de denunciar las desigualdades vigentes con miras a lograr transformaciones políticas. Al respecto Arendt plantea que "el poder se deriva básicamente de la capacidad para actuar en común, este poder descansa sobre convicciones, sobre una coacción no coactiva con que imponen las ideas y que se regula mediante un vínculo institucional reconocido" (2001: VII).

En el caso de las mujeres negras a través de la historia han desarrollado diferentes formas de resistirse. Durante la época de la colonia se aplicó el cimarronaje doméstico siendo aquellas acciones solapadas o expresas protagonizadas por las esclavas, destinadas a burlar el poder del amo (Hernández, 1995: 9). Aunado a lo anterior enfrentaron directamente al sistema político y judicial hasta que fueran reconocidos derechos que desde el sistema nunca se habían considerado para ellas y su grupo racial (Acuña, 2004: 13; 2).

Por otra parte se dio solidaridad de grupo, ante lo cual Acuña plantea que al tener una historia común las personas negras desarrollaron una conciencia de grupo. Asimismo, se dio el establecimiento de redes familiares, en tanto las mujeres negras en Costa Rica hicieron un gran esfuerzo para ayudarse mutuamente $y$ mantener la comunicación de sus afectos (1999: 8).

También las mujeres negras mantuvieron las estrategias de sobrevivencia y su legado étnico-racial por medio de la tradición oral, trasmitiendo su historia de generación en generación (1999: 8), con lo cual se evidencia cómo en la actualidad en las mujeres negras prevalece una posición de lucha y cambio social, siendo la continuidad de las ideas trasmitidas de mujer a mujer a través de la historia.

\section{PROCESO DE LA INVESTIGACIÓN}

El tipo de investigación que se desarrolló fue cualitativa al propiciarse un "proceso de descubrimiento del conocimiento desde las acciones humanas y la vida social, el interés es conocer la conducta humana desde el propio marco de referencia de quien actúa para así generar teoría" (Barrantes, 1999: 70). El estudio se basó en las vivencias de un grupo de mujeres afrocostarricenses, a partir de lo cual se puede dar cuenta de la realidad social, por lo que se destaca la participación activa de las informantes como sujetas de la investigación.

Asimismo, como fundamento epistemológico se retomó el feminismo en tanto propone teorías del conocimiento alternativas, las cuales consideran a las mujeres como sujetas del conocimiento (Harding, 1998: 14). Al respecto De Lauretis plantea:

Los conocimientos producidos por los estudios feministas han estado reconstituyendo a las propias mujeres como sujeto social, como sujeto del conocimiento y sujeto cognosciente simultáneamente, [por ende] se han definido marcos epistemológicos como consecuencia de la afirmación de la oposición (1986: 168) [en este caso las mujeres negras].

De esta manera se propuso "empezar por la vida de las mujeres" (Harding, 1998: 21) de ahí que la investigación haga alusión a sus propias 
voces para contar una realidad superando los discursos desde el sistema patriarcal vigente, los cuales se han definido como la historia oficial.

Por otra parte se consideró la tensión dialéctica entre las actoras sociales involucradas: la investigadora y el grupo de mujeres negras que participaron. Se retoman a Molina y Romero (2001) quienes plantean que cada agente tiene sus concepciones, historia, ubicación social, por lo que el trabajo se constituye en una fusión de espacios comunes, considerando también las diferencias que las caracterizan. En la presente investigación se comparte el hecho de ser mujer como constructo social en la sociedad costarricense y se consideran las múltiples diferencias basadas en las características raciales, lo cual se valora, retomando a Braidotti (2000) en vez de una desventaja como una posibilidad de integralidad del proyecto.

En relación con las sujetas facilitadoras de la información, el trabajo de investigación se realizó con 28 mujeres afrocostarricenses, residentes de las provincias de Limón y San José. Las mismas oscilaron en edades entre los 18 y los 79 años, específicamente cinco jóvenes adultas de 18 a 25 años; seis adultas jóvenes de 26 a 35 años; diez adultas medias de 36 a 59 años $y$ siete adultas mayores de 60 años en adelante.

En el nivel educativo los grados de estudio de ellas son variados, diez de ellas tienen estudios universitarios completos, cinco de ellas estudios especializados, dos tienen la secundaria completa, cuatro la secundaria incompleta de las cuales tres continúan estudiando en este nivel y siete primaria completa.

Se intentó identificar un grupo variado en cuanto a edades, ocupaciones, nivel educativo, estado civil y creencias religiosas, siendo el elemento determinante el que se auto identificaran como mujeres negras por tener ascendencia negra, por sentirse mujeres negras según su propia historia de vida $y$ por tener un ligamen étnico-racial.

El proceso de investigación se realizó en tres momentos. La etapa preparatoria, donde se contempló la revisión y el análisis bibliográfico a nivel teórico e histórico, para lo cual se definieron cinco variables que permitieron organizar la información: la esclavitud como fenómeno socio-histórico y sus representaciones actuales; la vivencia de la esclavitud en las mujeres negras; la situación actual de las mujeres afrodescendientes en América Latina y Costa Rica; el sexismo y el racismo y Epistemología y género.

En un segundo momento, se llevó a cabo el trabajo de campo, para lo cual también se definieron variables generales con el fin de guiar el proceso de recolección de la información según los objetivos de la investigación, tales como: las formas de discriminación y opresión como representaciones contemporáneas de la esclavitud; las vivencias de las mujeres afrodescendientes según sus sentimientos, percepciones y acciones; las estrategias de organización individual y colectiva de las mujeres afrodescendientes e información general de las facilitadoras.

La conformación del grupo de mujeres participantes se inició a través del "Centro de Mujeres Afrocostarricenses”, quienes facilitaron los primeros contactos. Posteriormente se aplicó el método de bola de nieve, con el fin de ampliar la representatividad del grupo, incluyendo mujeres de tres agrupaciones ligadas al Centro, de una agrupación comunal independiente $y$ mujeres que no participaban de ninguna agrupación.

Como técnica principal se aplicó la entrevista a profundidad, para lo cual se elaboró una guía a partir de las variables previamente definidas. Dicho instrumento fue sujeto a cambios durante el proceso de investigación según la información que fueron proporcionando las participantes al definirse algunos aspectos que se consideraron importantes ahondar. Asimismo, la aplicación de la guía de entrevista a profundidad se amoldó según la dinámica que se generó con cada participante, intentando cubrir todos los aspectos de interés para la investigación, pero ante todo se destacó la importancia de proporcionar un espacio para escuchar experiencias significativas para las mujeres, como vivencias específicas de cada una de ellas que reflejen su sentir por su raza y género.

Al iniciar cada proceso de entrevista se aplicó el protocolo de consentimiento informado en el que se contempló la identificación de la facilitadora, la descripción del proyecto y de la 
institución bajo la cual se estaría realizando la investigación, los objetivos de la investigación, se les consultó sobre su interés por participar y si existía de su parte algún tipo de restricción $y / o$ pregunta.

Como otras fuentes de información se realizaron entrevistas abiertas con expertos $y$ expertas en temas relacionados con la población afrocostarricense y líderes comunales, para lo cual se contemplaron las siguientes variables: las formas de sexismo y racismo actuales en Costa Rica; la historia de la población afrocostarricense enfatizando en la esclavitud y sus representaciones y la organización social de esta población en especial de las mujeres negras.

La información obtenida en las entrevistas fue grabada, lo cual fue consultado previamente con cada una de las personas participantes. Posteriormente se transcribió y organizó por temas, permitiendo el análisis de los datos.

Para dicho análisis se inició un proceso de contraste de la revisión bibliográfica en relación con la historia de las mujeres afrocostarricenses con las vivencias manifiestas por las mismas en la actualidad, aunado a lo anterior los resultados fueron reflexionados de una manera crítica desde la teoría feminista.

Asimismo, la información fue validada a través de la consulta con seis mujeres afrocostarricenses, quienes además de contar con vivencias como mujeres negras en Costa Rica, han desarrollado investigación y participado a un nivel formal en la organización y desarrollo de la población afrodescendiente en Costa Rica, a través de instituciones y organizaciones tales como la Universidad de Costa Rica, Proyecto Caribe, El Centro de Mujeres Afrodescendientes, La Organización Negra Centroamericana (ONECA), La Red Afroamericana y la Red de Abogados Afrolatinoamericanos.

\section{VIVENCIAS DE LAS FORMAS CONTEMPORÁNEAS DE LA ESCLAVITUD EN LAS MUJERES AFROCOSTARRICENSES}

\subsection{FORMAS DE DISCRIMINACIÓN Y OPRESIÓN}

A partir de la investigación realizada se logró identificar que el $100 \%$ de las mujeres afrocostarricenses consideran que en Costa Rica sí existe discriminación hacia la población negra en general $y$ por ende hacia las mujeres negras. Al respecto 19 de las facilitadoras afirmaron haber vivido directamente discriminación como mujeres afrocostarricenses y 9 de las mismas realizaron tal afirmación porque lo han vivido de manera indirecta, es decir por experiencias de personas cercanas o porque lo han observado en la dinámica social. Sin embargo, en este último grupo en los relatos subsecuentes de 7 de las mujeres, se identificó que sí han vivido situaciones de discriminación y opresión directas.

En general se encontró una tendencia mayoritaria de valorar estas experiencias como hechos superados por ellas $y$ de realizar una reflexión positiva, esto a pesar de que identifican que tal problemática ha repercutido en sus vidas.

Asimismo, algunas de las mujeres negras expresaron sentirse menos discriminadas por su color de piel, en tanto para el común de los/as costarricenses son identificadas como "mulatas" por tener un color de piel más claro, más allá que por su etnia. Por ende, se evidencia como directamente las experiencias de discriminación se maximizan o no desde su cuerpo físico, específicamente el color de piel, en tanto es simbolizado negativamente por estereotipos $y$ prejuicios vigentes.

El $100 \%$ de las mujeres participantes informaron sentirse mayormente discriminadas como mujeres negras en relación a los hombres de su mismo grupo racial, percibiendo que existe mayor aceptación hacia el hombre negro por parte de la población mestiza costarricense.

Por otra parte, dentro de las experiencias narradas por las facilitadoras se identificó que las expresiones de discriminación y opresión hacia ellas se dan en diferentes ámbitos sociales a un nivel formal e informal. Asimismo, afirmaron que este tipo de discriminación se presenta mayormente de manera indirecta, es decir no confrontativa ya que formalmente se niega esta realidad.

A continuación se agruparon las principales manifestaciones de discriminación y opresión por el género y la raza hacia las mujeres negras en Costa Rica, contemplando los relatos 
comunes que expresaron las facilitadoras de la información y relacionándolo con las formas en que se aplicó el sistema esclavista en las mujeres afrocostarricenses.

\section{$\diamond \quad$ EL LENGUAJE}

Algunas de las facilitadoras perciben que se da una diferenciación social desde el uso de la palabra "negra" como sustituto de sus nombres, siendo una forma de ubicarlas en un grupo social considerado inferior por su raza $y$ género $y$ ocultando su identidad primaria, el nombre. Asimismo, perciben esta palabra como una manera de acercamiento principalmente por parte de hombres desconocidos como un trato sexualizado hacia ellas.

Al respecto manifestaron que se da una identificación diferente por parte de las personas mestizas hacia los hombres de su mismo grupo racial, llamándolos "Señor" o por su propio nombre, al igual que hacia las mujeres mestizas, a quienes llaman como "muchacha", "señora" o también por su nombre, denotando con esto un mayor respeto.

Acuña (1999) plantea como desde el sistema esclavista, en Costa Rica, las mujeres negras dejaron de ser Ana, Rosa, Petronila por "la negra", palabra que contenía los significados negativos atribuidos a ellas para justificar los maltratos a los que fueran sometidas.

... a mí por ejemplo me ha pasado que yo voy, yo ando en la calle con mis amigas, a mis amigas les pueden decir señora o muchacha y a mí me dicen negra... Jenny ${ }^{1}$

Lo anterior no quiere decir que con esta denuncia las mujeres negras se encuentran haciendo una negación de su raza, sino que es una manera de hacer notar un trato desigual, lo cual perciben también en el uso de frases comunes que reflejan estereotipos que prevalecen, de los cuales son víctimas por parte de personas

Con el fin de proteger la identidad de las mujeres facilitadoras de la información se utilizarán pseudónimos. extrañas, de sus propias familias $y$ de lugares formales como lo son sus centros de trabajo.

\section{$\diamond \quad$ ABUSOS DIRECTOS}

Algunos de los relatos de las mujeres participantes fueron en relación con abusos directos que han vivido a nivel extrafamiliar, como insultos, empujones y burlas de personas mestizas, quienes han hecho alusión a su raza y género para fundamentar sus acciones de agresión. De alguna manera se puede inducir que el hecho de que existan personas que se den esta autorización es porque prevalecen ideas fundamentadas en la misma esclavitud como sistema que precisamente legalizó estas acciones.

... estaba en el bus e iba subiendo cuando un señor me jaló y me sacó de la puerta... me bajó... yo reaccioné y viene negra indecente, negra indecente... es que no tiene el derecho... Ligia

\section{$\diamond \quad$ EL BLANQUEAMIENTO SOCIAL}

Esto lo perciben como una tendencia de los hombres negros por preferir mantener relaciones de pareja formales con mujeres "blancas" refiriéndose a extranjeras con mayores recursos económicos y mujeres mestizas.

A pesar de lo anterior, esta situación no es percibida por la mayoría de las mujeres como un rechazo hacia ellas, sino como una manera de que los hombres de su mismo grupo racial buscan para tener ganancias segundarias en tanto prestigio social y recursos económicos. Asimismo, como una alternativa utilizada por los hombres de su mismo grupo racial ante el patriarcado vigente, ya que "pueden ejercer dominio sobre una mujer por cuanto ellas no se los permiten". Por ende, esta situación ha sido resignificada por las mujeres negras como una forma de empoderamiento desde el género.

\section{$\diamond \quad$ OPORTUNIDADES LABORALES}

Como parte de algunos de sus relatos expresaron tener dificultades en cuanto a las oportunidades laborales, esto fue una 
experiencia identificada independientemente del nivel educativo de las mujeres. Las facilitadoras contaron experiencias donde directamente les negaron trabajos por su color de piel o al ser contratadas fueron sujetas de diferentes formas de discriminación.

Se puede afirmar que las mujeres negras al nivel laboral pasan por un doble filtro social, primero superar una serie de prejuicios para que logren obtener un puesto como parte del grupo racial afrodescendiente $y$ demostrar sus capacidades como mujeres, tanto para la contratación como durante el desempeño de su trabajo, percibiendo que están siendo probadas de manera constante.

$\mathrm{Al}$ respecto la mayoría considera que los hombres de su mismo grupo racial tienen más oportunidades que ellas, aunque tengan experiencia y conocimientos inferiores.

Por otra parte, dentro de algunos de los relatos informaron que se limitan sus posibilidades laborales en funciones donde se "dé la cara” ante el público, asimismo, mayores posibilidades en trabajos privados, en tanto sean lugares donde no tengan comunicación directa con otras personas. En este caso se intensifican los mandatos del patriarcado donde se reserva la mujer al ámbito privado del hogar y donde se simboliza lo negro de manera negativa.

El tema de las oportunidades laborales también denota cómo la discriminación y opresión hacia las mujeres negras va más allá de las relaciones personales, se instala en espacios formales y a un nivel macrosocial, es decir no responde a percepciones o decisiones específicas de personas mestizas, sino que son ideas legitimadas como parte de toda una estructura social.

\section{$\diamond \quad$ DISCRIMINACIÓN MACROSOCIAL}

En algunas de las experiencias relatadas por las mujeres negras entrevistadas, retomaron formas de discriminación y opresión que las han afectado directamente al estar insertas como parte de las políticas institucionales e incluso en la legislación vigente del país, dentro de las cuales reconocen abusos de poder hacia la población negra en su caso por cuestiones raciales $y$ sexistas.
Dentro de los temas que mencionaron se encuentran políticas en cuanto a la adjudicación de tierras, formas de organización de las asociaciones comunales, el establecimiento de penas carcelarias y otras resoluciones judiciales y el tipo de programas en la educación pública.

... todavía cuando yo nací me pusieron como Jamaiquina... mi papá y mi mamá nacieron aquí pero como les pusieron Jamaiquinos entonces a mí también. Hellen

\subsection{MECANISMOS DE RESISTENCIA DE LAS MUJERES AFROCOSTARRICENSES}

A partir de la revisión bibliográfica de la historia de las mujeres negras en América Latina y específicamente en Costa Rica, durante la época de la esclavitud formal como en las subsiguientes representaciones de la esclavitud $y$ retomando el apartado anterior en el que se intentó sintetizar sus vivencias actuales de discriminación y opresión por el género y la raza, surge la pregunta ¿de qué manera las mujeres negras, ante la opresión por el género y subordinación por su raza, han logrado sobrevivir $y$ además obtener avances significativos en su situación legal, trato social y condiciones de vida para ellas, para sus familias y para su pueblo en general, teniendo repercusiones de cambios paulatinos en la sociedad hasta en la época contemporánea? Desde la presente investigación se analizaron algunas de sus estrategias.

\section{$\diamond \quad$ «OY NEGRA"}

Al respecto se contempla la autopercepción de las mujeres negras haciendo una resignificación de su raza, es una manera de resaltar su color de piel frente a los otros, mestizos/as, como una diferenciación positiva.

$\mathrm{Al}$ respeto Campbell manifiesta:

... género y etnia son indivisibles en nuestros planteamientos, propuestas 
$y$ denuncias. Nos autodefinimos como mujeres negras, que han sido las características para nuestra exclusión y será como mujeres negras que impulsemos una propuesta... (2002: 32).

Dentro del grupo de mujeres entrevistadas, el hecho de definirse como mujeres negras representa superar características adjudicadas a las mujeres desde una ideología patriarcal, comparándose con las mujeres mestizas.

Estas características las relacionan directamente con sus historias personales y con las vivencias de sus ancestras como mujeres que lucharon. De esta manera se identifica que ellas entienden su historia como un motor para buscar el cambio hoy.

... somos mujeres muy fuertes, aprendemos muchas cosas, sobrevivimos muchas cosas también, no nos echamos a la borda así tan rápido. Scarlett

\section{$\diamond \quad$ FORTALEZA ESPIRITUAL}

Varias de las mujeres identificaron la creencia en un Dios, como una estrategia que les ha permitido enfrentar situaciones concretas donde han vivido discriminación sin llegar a sentirse inferiores por su color de piel, asimismo al enfrentar a personas no permitiendo que las maltraten.

Al respecto el espacio de la iglesia se ha convertido en una forma de congregarse como población afrocostarricense, siendo las mujeres negras quienes han asumido el liderazgo. Consecuentemente han trascendido a la organización comunal para discutir y movilizarse ante problemas que les aqueja como grupo racial y desde su género.

... es una manera de organizarnos, de hacer algo, porque ir a la iglesia a cantar a Dios y volver a la casa no es suficiente... con el fin de levantarnos la autoestima, de conocernos mejor, de apoyarnos en todas las áreas de nuestra vida. Betty

\section{$\diamond \quad$ LA LIBERACIÓN DE LA MENTE}

Al respecto se identificó como liberación de la mente a aquellas reflexiones personales expresadas por las facilitadoras que les permiten tener una actitud positiva frente a circunstancias adversas a su alrededor.

... a mí nunca me van a esclavizar, aunque yo pudiera tener unas cadenas acá no me van a esclavizar, en mi corazón no... Anabelle

\section{$\diamond \quad$ UNA POSICIÓN DE LUCHA}

En los discursos de las mujeres negras se identificó de manera constante el que se identificaran en "una lucha continua". Dentro de esto contemplan la detección de todas las formas de discriminación y opresión en la sociedad, hasta las formas más sutiles, con el fin de denotarlas y transformarlas. Esta resistencia refleja que en general prevalece un activismo y compromiso social.

Lo que tengo es por la lucha que yo misma pude dar, en un país donde soy minoría y donde tenía todo en contra. Marlen

\section{$\diamond \quad$ ORGANIZACIÓN SOCIAL}

Esta inicia desde el ámbito familiar en donde las mujeres negras han asumido la tarea de reproducir las costumbres y tradiciones, así como valores $y$ creencias, siendo estrategias de fortalecerse y autoafirmarse como grupo étnico-racial.

Aunado a esto se percibió cómo el 100\% de las mujeres participan en diferentes grupos sociales y comunales que tienen algún objetivo explícito o indirecto para congregarse con el fin de mejorar las condiciones negativas que enfrentan como mujeres negras. Esto es independiente de la edad y grado académico de las facilitadoras, con lo que se evidenció la intención de organizarse dentro de las posibilidades de cada una al prevalecer la alternativa de salir $y$ hacer algo por ellas como grupo, a pesar de que no sea de manera directa, confrontativa, formal o reconocida. 
Dentro de los grupos en los que participan nombraron cursos de aprendizaje donde enfatizan la transmisión de sus conocimientos culturales, grupos de desarrollo comunal y social, grupos de la iglesia donde por medio de una visión espiritual de la vida promueven el autorrespeto, la valoración personal como mujeres negras y la fortaleza personal ante la misma discriminación y opresión como mujeres $y$ negras. Asimismo se identificó la participación de algunas de las mujeres en organizaciones más formales, con representación política y en instancias internacionales.

\section{$\diamond \quad$ ELMESTIZAJE}

Dentro de las experiencias de las mujeres negras se identificó que algunas de ellas, aunque una minoría, utilizan el mestizaje para contrarrestar situaciones de discriminación $y$ opresión social por su raza y por su género.

Algunas de las participantes identificaron esta estrategia por lo observado en otras mujeres de su grupo racial; sin embargo, expresaron no aplicarlo ni estar de acuerdo. Tal situación no se relaciona con el grado académico de la mujer, más bien se identificó como una decisión según sus experiencias de vida.

Específicamente una minoría expresó no desear relacionarse al nivel de pareja con alguna persona considerada por ellas como "más negra" según el color de piel. Lo anterior lo relacionan directamente con la maternidad al pensar en tener hijos/as con un color de piel más oscuro, ante lo cual pudieran sufrir una mayor discriminación.

A pesar de lo exteriorizado en relación con las características físicas, en general manifestaron desear mantener y fomentar las costumbres étnicas con sus hijos/as, por lo que no perciben el mestizaje como una negación de la cultura afrocostarricense.

\section{$\diamond \quad$ LAEDUCACIÓN FORMAL YAUTODIDACTA}

De las mujeres negras que participaron en la investigación la gran mayoría expresó la necesidad de estudiar para ascender social- mente, como una forma de responder a la discriminación y opresión que se mantiene en la sociedad costarricense y por ende en sus discursos, repitieron constantemente la idea de aprovechar las oportunidades.

$\mathrm{Al}$ respecto sus discursos son coincidentes con estadísticas que reflejan la realidad de las mujeres negras, ya que según Putnam (2000) las mujeres afrocostarricenses que realizan estudios universitarios proporcionalmente superan a cualquier grupo étnico incluso a los hombres de su mismo grupo racial.

Esto va más allá de la educación formal, ya que incluso en las zonas rurales y en el caso de mujeres con recursos económicos limitados se identificó como buscan tener opciones de aprendizaje ya sea formales $\mathrm{u}$ organizadas por ellas mismas, apoyándose de manera solidaria entre sí. Asimismo, definen esta opción para su desarrollo personal como para incentivar tal práctica en su descendencia y comunidad.

\section{$\diamond \quad$ EL RESCATE DE SU FEMINIDAD FRENTE A UNA RESIGNIFICACIÓN DE SU PAPEL FEMENINO}

Como se mencionó en el capítulo anterior, las mujeres negras viven situaciones de discriminación y opresión específicas por su raza y género, en el ámbito personal y en el social mayor. Es así como ante dichas vivencias ellas mismas desarrollan formas de resistir y protegerse siendo específicas en su identidad de mujeres negras.

Como parte de este aspecto, también se puede rescatar desde una interpretación de sus discursos, una resignificación de su feminidad en cuanto al aspecto sexual se refiere.

Ante la sexualización vigente a la que han estado expuestas y retomando la historia de su ancestras, algunas expresaron la importancia de remarcar límites ante las otras personas, principalmente los hombres en cuanto a los prejuicios sexuales a su alrededor, percibiéndolo como un respeto hacia sus cuerpos.

Asimismo, por otra parte, defender costumbres que ante la población mestiza pueden tener un significado sexual pero que para ellas representan maneras de expresarse que las agrupa e identifica. Estas han sido trasmitidas 
de generación en generación, tales como los bailes y un control consciente de la reproducción.

Otra forma de resignificar lo femenino a través de la identidad racial, ha sido incorporar actividades $y$ actitudes no tradicionales para las mujeres según el sistema patriarcal, siendo identificado por ellas como un legado trasmitido por la vía materna. Estas actitudes y actividades que se consideran trascienden la división binaria de las personas según sus sexos, es vista por ellas como una diferenciación positiva frente a las mujeres mestizas, por ende su raza, su historia, su legado les ha permitido empoderarse como mujeres y colectivo social.

\section{CONCLUSIONES}

El proceso de la investigación permitió retomar los relatos de un grupo de mujeres negras costarricenses en cuanto a sus vivencias de discriminación y opresión por factores raciales $y$ de género, $y$ constituyó un medio para replantear los discursos oficiales frente a su realidad, desde sus propias voces. Al respecto se retoma a Tubert (1995) quien rescata la necesidad de construir representaciones discursivas diferentes como mecanismo de derrocamiento de las mujeres.

La información proporcionada por las mujeres participantes de la investigación, permitió conocer experiencias que reflejaron la manera en que se reproducen ideologías basadas en principios raciales y como parte de todo un sistema patriarcal vigente.

Al respecto se constató primeramente que la esclavitud como sistema socioeconómico formal fue abolida legalmente; sin embargo, las ideas que la fundamentaron continúan vigentes en la sociedad costarricense. Si bien es cierto estas ideas se han representado a través del tiempo en prácticas análogas a la esclavitud hasta en la actualidad, lo cual se puede analizar de manera más profunda en un estudio posterior; se dio énfasis a la manera en que continúan dándose como una mentalidad instalada y que se manifiesta tanto en un nivel simbólico como en prácticas de discriminación y opresión concretas.
Asimismo, la discriminación de la que son objeto va más allá de su etnia, se justifica directamente en sus cuerpos, específicamente por su color de piel, ya que en tanto mayor mestizaje físico menor discriminación. A la vez va más allá de la raza en la medida que son objeto de mayores discriminaciones por su género.

Específicamente se identificó que las formas de discriminación y opresión se reproducen al nivel informal, es decir en las relaciones sociales cotidianas, como al nivel formal, en prácticas institucionales.

Lo anterior se gesta primeramente desde el lenguaje, por tanto las palabras se constituyen en un medio inmediato que reflejan las ideas de las personas, en este caso en el común de los/as costarricenses y por lo tanto en la dinámica que prevalece en la sociedad.

Consecuentemente se evidenciaron abusos directos que viven las mujeres negras, implicando maltrato físico, verbal y prácticas de sexualización mayores en relación con las mujeres de otros grupos raciales. En este ámbito se retoma el concepto de confinamiento simbólico propuesto por Bourdieu (2000), ya que el caso de las mujeres negras se representa con mayor fuerza en la exigencia social del deber ser determinado por el género en cuanto a la manera en que se espera deben presentarse y actuar, lo cual sin duda se relaciona con la sexualización vivida por sus ancestras esclavas.

También las representaciones de esclavitud contemporáneas que viven las mujeres negras en Costa Rica se evidencian a través de la práctica del blanqueamiento social, comprendiéndolo como el mestizaje social y racial utilizado por un sector de la población masculina de su grupo racial.

Asimismo, se comprobó que continúan teniendo limitaciones en cuanto a las oportunidades laborales $y$ de desarrollo personal, esto al negárseles trabajos o ser evaluadas doblemente para demostrar sus capacidades, lo cual viven de manera más rígida en relación con los hombres de su grupo racial y a otras mujeres. Aunado a lo anterior, se identificaron formas de discriminación racial $y$ de género a un nivel macrosocial en cuanto a normativas institucionales y legales tácitas. 
La discriminación por el género es determinante de las formas de discriminación por la raza, en tanto ambas características se contraponen teniendo una realidad específica como mujeres negras. Por ende prevalecen las formas de discriminación que viven ellas en relación con las mujeres mestizas y a los hombres negros.

Además el tipo de discriminación que viven las mujeres negras en la actualidad, se relaciona directamente con la historia de sus ancestras esclavas. Si bien es cierto actualmente no son abusadas sexualmente sin restricciones como sí lo vivieron sus antecesoras esclavas, según las vivencias expresadas por las mujeres que participaron en la investigación, muchas de ellas han sido tratadas por algunas personas como objetos sexuales y según su percepción esto se da de manera más fuerte hacia ellas que hacia mujeres de otros grupos raciales. Si ya no son explotadas laboralmente se les dificulta mayormente por su color de piel obtener un trabajo y optar por actividades no tradicionales para las mujeres; si ya no son objeto de todo un sistema de mestizaje y estratificación social legal instalado a partir del color de piel de las personas, se continúan dando estrategias de blanqueamiento social.

Sin embargo, se puede afirmar que paradójicamente la historia de esclavitud de sus ancestras las ha fortalecido desde sus propias historias y como colectivo de mujeres negras. A través de la tradición oral como estrategia de las mujeres negras esclavas para derrocar la esclavitud, ha prevalecido la idea de lucha, llevándolas a establecer resistencias individuales como redes comunales y organizaciones formales.

Específicamente se denota la resignificación de lo negro para una deconstrucción de su papel femenino. Su raza, en vez de representar para ellas una desventaja ante el trato social que viven, ha sido valorada como una diferencia como grupo específico para superar mandatos de género en relación con otras mujeres, lo cual también se gesta desde el conocimiento y legado de la realidad vivida por las mujeres negras esclavas.

Como estrategias de resistencia utilizadas se identificaron la autodefinición y rea- firmación social como mujeres negras para la diferenciación positiva en cuanto a la exigencia de sus derechos; la utilización de recursos espirituales como legado étnico-racial a cargo de las mujeres, lo cual a su vez les ha facilitado la utilización de recursos personales para sobrevivir a las dificultades, siendo lo que se denominó como "la liberación de la mente" y se ha convertido en una base para la congregación de su comunidad y la organización como mujeres ante los problemas que las aquejan.

Asimismo, se identificó "una posición de lucha" contemplando su compromiso personal $y$ grupal por denunciar las diferentes formas de discriminación y opresión que se continúan reproduciendo en la sociedad costarricense. A su vez utilizan medios formales para su desarrollo personal como lo son la educación formal, autodidáctica y redes de apoyo comunal, incluso han trascendido a desarrollar toda una organización formal como colectivo de mujeres siendo transversal a los diferentes estratos socioeconómicos.

En todo este proceso de resistencias como mujeres negras sin duda la historia ha sido un elemento determinante, más que para el trato social que viven por prejuicios de género y raciales vigentes, para activar una transmisión entre mujeres de una posición de lucha, a través de distintas generaciones y realidades sociales. Retomando a Campbell (2003) sin duda "las mujeres negras construyen la historia”.

\section{BIBLIOGRAFÍA}

Acuña, María de los Ángeles. "Mujeres esclavas en la Costa Rica del siglo XVIII: estrategias frente a la esclavitud". [Tesis de Doctorado en Historia]. Universidad de Costa Rica.1999.

Acuña, María de los Ángeles. "Costa Rica colonial: roles productivos $y$ reproductivos de las mujeres esclavas". [VIII Congreso Centroamericano de Historia]. Honduras, 2004. 1. 
Acuña, María de los Ángeles. "Explotación y resistencia: el mundo de las mujeres esclavas en la provincia de Costa Rica en el siglo XVIII". [Ponencia VII Congreso Centroamericano en Historia]. Honduras, 2004. 2.

Amorós, Celia (1985). Hacia una crítica de la razón patriarcal. ¿Feminismo existencialista versus feminismo estructuralista? España: Ed. Anthropodos, 1991.

Arendt, Hannah (1974). La Condición Humana. España: Editorial Paidós, 2001.

Barrantes, Rodrigo. Investigación: Un cambio al conocimiento, un enfoque cuantitativo $y$ cualitativo. Costa Rica: Editorial EUNED, 1999.

Bastide, Roger. El prójimo y el extraño. El encuentro de las civilizaciones. Argentina: Editorial Cujans, 1970.

Bourdieu, Pierre. La Dominación Masculina. España: Editorial Anagrama, 2000.

Braidotti, Rossi. Sujetos Nómades. Argentina: Editorial Paidós, 2000.

Calvo, Silvia. "Formas contemporáneas de la esclavitud y prácticas análogas". [Tesis de Licenciatura en Derecho]. Universidad de Costa Rica. 2004.

Campbell, Epsy. Poderes cuestionados: sexismo y racismo en América Latina. México: UNIFEM, 2002.

De Beauvoir, Simone (1972). El Segundo Sexo. Argentina: Editorial Sudamericana, 1990.

De Lauretis, Teresa (1986). Estudios Feministas/estudios críticos: problemas, conceptos y contextos. México:
Universidad Autónoma Metropolitana, 1996.

Harding, Sandra (1986). ¿Existe un método Feminista? España: Editorial Morata, 1998.

Hernández, Omar y otros. Discriminación y racismo en la historia de Costa Rica. Costa Rica: Editorial de la Universidad de Costa Rica, 1993.

Irigaray, Luce (1990). Amo a Ti. El otro: mujer. Argentina: Ediciones La Flor, 1994.

Lamas, Marta (1996). "Usos, dificultades y posibilidades de la categoría 'género"”. Lamas, Marta (comp.). El Género: la construcción cultural de la diferencia sexual. México. Pueg/Porrúa, 1996: 327367.

Molina, Lorena y Romero, María Cristina. Modelos de intervención asistencial, socioeducativa y terapéutica en Trabajo Social. Costa Rica: Editorial de la Universidad de Costa Rica, 2001.

OACDH. Convención Suplementaria sobre la Abolición de la Esclavitud, la Trata de Esclavos y las Prácticas Análogas a la Esclavitud. Ginebra, 1956. [Documento en Línea] En: <http://www.ohchr.org/ spanish/law/abolicion.htm $>$ [consultado 28 de septiembre 2009].

OACDH. "Formas contemporáneas de la esclavitud". Folleto Informativo 14. Suiza, s/f. [Documento en Línea] En: <http://www.ohchr.org/Docuemnts/ Publications/FactSheet 14 sp.pdf $>$ [consultado 28 de septiembre 2009].

Putnam, Lara. La población Afrocostarricense según los Censos del 2000. Costa Rica: INEC, 2002. [Documento en Línea] En: <http://www.inec.go.cr/ INEC_DIS/Publicaciones/archivo\%20 
SerieCensal\%20xls/pob\%20afrocost.pdf> [consultado 28 de septiembre 2009].

Tubert, Silvia. Psicoanálisis y Feminismo. España: Editorial Cátedra, 1995.
Velzeboer, Marijke y otras. La violencia contra las mujeres: responde el sector de la salud. Estados Unidos: ops, 2003. [Documento en Línea] En: $<$ http://www.paho.org/Spanish/AD/ GE/VAW-HealthSectorRespondsSP.pdf> [consultado 28 de septiembre 2009]. 